\title{
A revolução tecnológica e os benefícios do marketing digital respaldados pela ética odontológica: revisão integrativa de literatura
}

The technological revolution and the benefits of digital marketing supported by dental ethics: integrative literature review

La revolución tecnológica y los beneficios del marketing digital apoyados en la ética dental: revisión bibliográfica integradora

\section{Resumo}

É possível usar a mídia digital tanto para atrair quanto para fidelizar pacientes, utilizando essa ferramenta para disseminar conhecimento, serviços e facilitar a comunicação entre cliente em potencial e clínica. Desse modo, é indispensável que o cirurgião dentista esteja ciente dos conceitos éticos, presentes no Código de Ética Odontológico, ao utilizar as ferramentas de publicidade e marketing para o benefício próprio, para assim trabalhar com coerência e segurança. O objetivo desse estudo é caracterizar os benefícios do marketing digital em odontologia, preconizando o Código de Ética Odontológico. Foi realizada uma revisão integrativa da literatura, com os descritores "Ethic", "Dentistry" e "Digital Marketing", associados pelos operadores booleanos "and" e "or", por meio do qual foram selecionados, a partir dos critérios de elegibilidade propostos na estratégia PRISMA ScR, 5 artigos para análise qualitativa, nas bases de dados Scielo, Pubmed e BVs. O marketing digital tem sido utilizado como estratégia de divulgação dos serviços odontológicos na contemporaneidade e a partir dessa análise, conclui-se que o marketing digital contribui fortemente para o sucesso do cirurgião dentista, podendo afetar consideravelmente para o seu sucesso profissional. No entanto, seu uso deve ser pautado em condutas consonantes com o Código de Ética Odontológico, sendo de grande importância o conhecimento do mesmo pelo cirurgião dentista.

Palavras-chave: Ética odontológica; Marketing de serviços de saúde; Odontologia.

\begin{abstract}
It is possible to use digital media to both attract and retain patients, using this tool to disseminate knowledge, services and facilitate communication between potential customers and the clinic. Thus, it is essential that the dental surgeon is aware of the ethical concepts, present in the Dental Code of Ethics, when using advertising and marketing tools for their own benefit, in order to work with consistency and safety. The aim of this study is to characterize the benefits of digital marketing in dentistry, preconizing the Dental Code of Ethics. An integrative literature review was conducted using the descriptors "Ethic", "Dentistry" and "Digital Marketing", associated by the Boolean operators "and" and "or", through which 5 articles were selected, from the eligibility criteria proposed in the PRISMA ScR strategy, for qualitative analysis, in the Scielo, Pubmed and BVs databases. Digital marketing has been used as a strategy to promote dental services in contemporary times, and from this analysis, we conclude that digital marketing strongly contributes to the success of the dental surgeon, and can considerably affect his professional success. However, its use should be guided by conduct consistent with the Code of Dental Ethics, and knowledge of it by the dental surgeon is of great importance.
\end{abstract}

Keywords: Dental ethics; Marketing of health services; Dentistry. 


\begin{abstract}
Resumén
Es posible utilizar los medios digitales tanto para atraer como para retener a los pacientes, utilizando esta herramienta para difundir conocimientos, servicios y facilitar la comunicación entre los clientes potenciales y la clínica. Así, es fundamental que el cirujano dentista conozca los conceptos éticos, presentes en el Código de Ética Dental, a la hora de utilizar las herramientas de la publicidad y el marketing en beneficio propio, para trabajar con coherencia y seguridad. El objetivo de este estudio es caracterizar los beneficios del marketing digital en odontología, preconizando el Código de Ética Dental. Se realizó una revisión bibliográfica integradora, con los descriptores "Ética", "Odontología" y "Marketing Digital", asociados por los operadores booleanos "y" y "o", a través de los cuales se seleccionaron, a partir de los criterios de elegibilidad propuestos en la estrategia PRISMA ScR, 5 artículos para el análisis cualitativo, en las bases de datos Scielo, Pubmed y BVs. El marketing digital se ha utilizado como estrategia de difusión de los servicios odontológicos en la época contemporánea y, a partir de este análisis, se concluye que el marketing digital contribuye en gran medida al éxito del cirujano dental, y puede afectar considerablemente a su éxito profesional. Sin embargo, su uso debe basarse en conductas coherentes con el Código de Ética Dental, siendo de gran importancia el conocimiento del mismo por parte del cirujano dentista.
\end{abstract}

Palabras-clave: Ética odontológica; Comercialización de los Servicios de Salud; Odontología.

\title{
1. Introdução
}

Assim como várias áreas da ciência, a Odontologia também passou por processos importantes de evolução e reconhecimento para figurar entre as mais importantes profissões de saúde. Existiram diversos momentos históricos demarcados através de publicações especificas sobre a área, sendo um dos mais importantes aquele feito por Pierre Fauchard, considerado o Pai da Odontologia, no século XVIII, intitulado Le Chirugien Dentiste - Au Traité des Dents, e ainda com a adaptação às descobertas e demandas tecnológicas e tendências de forma insistente até os dias atuais (Martins, Dias e Lima, 2018).

Não só a Odontologia foi, historicamente, reconhecida anos depois do seu surgimento como uma ciência, mas o marketing também levou anos para ter a sua atividade oficializada, a partir de 1900, mesmo estando tão atrelado ao comércio, que é uma das mais antigas atividades humanas. Antes utilizado nas trocas, que são as primeiras formas de mercar descritas na história, hoje o marketing é aplicado nas mais diversas áreas ligadas à venda de produtos ou serviços como uma maneira de se estudar e analisar o mercado para que se possa satisfazer as necessidades do consumidor (Ayres Pinto et al., 2020).

No início da década de 70 o Departamento de Defesa dos Estados Unidos (DARPA), criou uma rede chamada ARPAnet, depois chamada de internet, sem saber que poucos anos depois, na década de 90 e com o surgimento dos computadores pessoais, ela seria usada mundialmente, mudando juntos, a forma de comunicação dos seres humanos. Isso tornou as fronteiras interpessoais e de serviços ao redor do mundo, menores (Rocha e Souza, 2016).

Nesse sentido, é possível perceber através dos anos a necessidade de o consultório odontológico ser visto como uma empresa e, assim, investir em formas de atrair e fidelizar os clientes, para o bem financeiro do empreendimento e consequentemente a entrega de um serviço de excelência para o paciente. Para isso, o marketing desenvolve um importante papel nesse ponto, sendo usado principalmente na esfera digital no século XXI e com o advento da internet, o marketing digital pode ser amplamente utilizado para alcançar esses objetivos. É possível usar a mídia digital tanto para atrair quanto para manter os pacientes, utilizando essa ferramenta para disseminar conhecimento, serviços e facilitar a comunicação entre cliente em potencial e clínica (de Lira \& Magalhães, 2018).

O uso das mídias sociais tem se tornado tão relevante na ciência odontológica que estudos bibliométricos como o de Pereira (2017) demonstram que o assunto tem sido amplamente discutido em periódicos de renome internacional com reputação consolidada, seja para a finalidade do uso destas ferramentas como estratégia de aprendizagem ou mesmo como recurso para o marketing digital. As redes sociais mais utilizadas de acordo com trabalhos como o de Kenny e Johnson (2016) no Estados Unidos e no Reino Unido são o Facebook, o Youtube e o Instagram, sendo o LinkedIn que tem a finalidade de relações profissionais entre as pessoas, o menos utilizado pelos cirurgiões dentistas e estudantes de Odontologia. No Brasil, 
números impressionantes sobre o uso das redes sociais também tornam estes redutos fundamentais para o marketing digital: são 102 milhões de brasileiros com acesso às redes sociais, o que torna essas ferramentas um recurso acessível e de amplo alcance para a publicidade e propaganda (Brasil, 2017).

Dentistas e estudantes de Odontologia acreditam que o marketing digital é mais abrangente e impactante do que o marketing convencional (Aboalshamat et al, 2019). Percebe-se que o conteúdo publicado nas redes sociais especialmente vinculado ao marketing odontológico no Facebook não está em consonância com o Código de Ética Odontológico (Oliveira, Prado e Silva, 2014), dessa forma dado o alto volume de uso das redes sociais por estudantes e profissionais de Odontologia e até por pacientes que se utilizam dos serviços prestados de saúde bucal, deve-se haver um maior controle tanto por parte de quem publica as ações publicitárias, tanto dos órgãos fiscalizadores e regulamentadores do exercício profissional da Odontologia (Garbin et al, 2018).

É indispensável que o cirurgião dentista esteja ciente dos conceitos éticos, presentes no Código de Ética, ao utilizar as ferramentas de publicidade e marketing para o benefício próprio, para assim trabalhar com coerência e segurança. O Código de Ética Odontológico (CEO) é de fácil acesso e leitura, porém, muitas vezes tem suas diretrizes burladas, por falta de fiscalização ou educação frente a sua utilização, fazendo com que exista concorrência desleal e propaganda irresponsável perante o paciente (Magalhães, 2018).

O objetivo desse estudo é caracterizar os benefícios do marketing digital em odontologia, preconizando o Código de Ética Odontológico.

\section{Metodologia}

O presente trabalho trata-se de uma revisão integrativa da literatura, a qual utilizou a estratégia PICO para elaborar a questão norteadora, em que, ao P (população) foi atribuído dentistas que buscam estratégias eficazes de marketing, I (intervenção ou indicador) uso das ferramentas digitais para marketing odontológico, C (comparação) estratégias de marketing não digital e $\mathrm{O}$ (outcome ou desfecho) estratégias éticas dentro do marketing digital como um diferencial no mercado de trabalho, obtendo assim a quentão norteadora: "O uso das ferramentas digitais seriam um diferencial no mercado de trabalho como uma estratégia ética e eficaz?”.

De acordo com as diretrizes PRISMA (Preferred Reporting Items for Systematic Reviews and Meta-Analyzes), a busca na literatura foi realizada, nas seguintes bases de dados: Scientific Electronic Library Online (SciELO) Web of Science e National Library of Medicine (PubMed/Medline), e Biblioteca Virtual em Saúde (BVS). Para a seleção dos artigos foram considerados os seguintes Descritores em Ciências da Saúde (DeCs) combinados com os operadores booleanos AND e OR: "Ethic", "Dentistry" e "Digital marketing".

Como critérios de inclusão para o estudo delimitaram-se artigos com período de publicação entre 2016 e 2021, com estudos que respondem à questão norteadora, com textos completos disponíveis online nos idiomas Inglês e Português e publicados nas referidas bases de dados. Para critérios de exclusão definiram-se: teses e dissertações, artigos fora do período de publicação definido, capítulos de livros e opiniões pessoais ou de especialistas.

\section{Resultados}

A busca de dados resultou em 42 artigos potencialmente elegíveis (Figura 1), dos quais 8 encontrados nas bases de dados US National Library of Medicine National Institutes of Health (PubMed Central), 21 artigos na Scientific Electronic Library Online (SciELO) e 13 artigos na Bibblioteca Virtual em Saúde (BVS). 
Figura 1 - Fluxograma do processo de seleção dos estudos primários.

Identificação

Seleção

Elegibilidade

Inclusão

$$
\begin{gathered}
\begin{array}{c}
\text { Registro identificado no banco de dados de } \\
\text { busca }(\mathrm{n}=42)
\end{array} \\
\downarrow \\
\text { Registro após eliminar os duplicados }(\mathrm{n}=0)
\end{gathered}
$$

$\downarrow$

\begin{tabular}{|l|l|}
\hline Registros selecionados $(\mathrm{n}=5)$ & $\rightarrow \quad \begin{array}{l}\text { Registros excluídos } \\
(\mathrm{n}=37)\end{array}$ \\
\hline
\end{tabular}

$\downarrow$

Artigo em texto completo avaliados para elegibilidade $(\mathrm{n}=5)$

Estudos incluídos em síntese qualitativa $(\mathrm{n}=5)$

Fonte: Autores.

A leitura dos títulos e dos resumos dos artigos foram os métodos utilizados para inclusão e exclusão, tendo sido eliminados 37 publicações. Ao final da análise alcançou-se a amostra final de 5 estudos primários. Os artigos excluídos não correspondiam durante a leitura de seu título ou resumo aos desfechos esperados nesta revisão, ou não se relacionavam ao escopo central dessa pesquisa.

Após elegibilidade dos artigos, eles foram organizados (Quadro 1) com extração dos dados baseada na ferramenta Mixed Methods Appraisal Tool (MMAT) (Galvão \& Ricarte, 2019), com a descrição do autor, ano de publicação, periódico, tipo de estudo e resultados. 
Quadro 1 - Caracterização do estudo, segundo autor principal, ano de publicação, periódico, objetivos, tipos de estudo, principais resultados e ferramentas digitais utilizadas.

\begin{tabular}{|c|c|c|c|c|}
\hline Autor/Ano & Periódico & Objetivo & Tipo de Estudo & Resultados \\
\hline Meira, 2021 & SpringerOpen & $\begin{array}{l}\text { Analisar a percepção do } \\
\text { público sobre a } \\
\text { credibilidade profissional } \\
\text { e a disposição para se } \\
\text { tornar cliente, a partir de } \\
\text { imagens postadas por } \\
\text { ortodontistas no } \\
\text { Instagram. }\end{array}$ & $\begin{array}{l}\text { Estudo } \\
\text { transversal }\end{array}$ & $\begin{array}{l}\text { Os ortodontistas e estudantes de odontologia } \\
\text { atribuem maior valor de credibilidade profissional } \\
\text { às imagens que ilustram aspectos técnicos, ao } \\
\text { contrário de leigos e especialistas de outras áreas } \\
\text { da odontologia. Temas relacionados à área } \\
\text { profissional geral do ortodontista e imagens de } \\
\text { antes e depois parecem ter maior influência na } \\
\text { percepção de credibilidade profissional, além de ter } \\
\text { maior impacto na conquista de futuros pacientes. }\end{array}$ \\
\hline $\begin{array}{l}\text { De Lira, } \\
2018\end{array}$ & $\begin{array}{c}\text { Brazilian } \\
\text { Dental Science }\end{array}$ & $\begin{array}{l}\text { Comparar o marketing } \\
\text { digital com as demais } \\
\text { estratégias de marketing } \\
\text { na odontologia } \\
\text { baseados no código de } \\
\text { ética, a fim de aumentar } \\
\text { a produtividade, captação } \\
\text { de novos pacientes e } \\
\text { fidelização daqueles em } \\
\text { tratamento. }\end{array}$ & $\begin{array}{l}\text { Revisão de } \\
\text { literatura } \\
\text { integrativa }\end{array}$ & $\begin{array}{l}\text { O marketing digital tem se mostrado de grande } \\
\text { importância no mercado de serviços odontológicos } \\
\text { se, em conjunto com as demais modalidades de } \\
\text { marketing externo e interno, obedecer aos preceitos } \\
\text { éticos do Conselho de Ética Odontológica. Há uma } \\
\text { grande aceitação do marketing digital pelos } \\
\text { cirurgiões-dentistas, tanto por meio de sites quanto } \\
\text { por meio das redes sociais, mas tem havido o } \\
\text { descumprimento dos preceitos éticos vigentes no } \\
\text { Código de Ética Odontológica }\end{array}$ \\
\hline $\begin{array}{c}\text { Guedes, } \\
2021\end{array}$ & $\begin{array}{l}\text { Rev. Rede de } \\
\text { Cuidados em } \\
\text { Saúde }\end{array}$ & 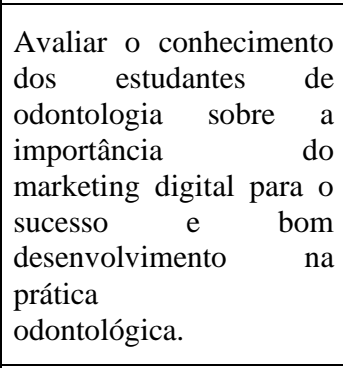 & $\begin{array}{c}\text { Estudo } \\
\text { Observacional } \\
\text { Tranversal }\end{array}$ & $\begin{array}{l}\text { O marketing digital pode ser um fator } \\
\text { determinante para o sucesso do cirurgião dentista } \\
\text { no seu cotidiano. Esse conteúdo deveria ser } \\
\text { empregado na grade curricular da graduação, já } \\
\text { que é considerado uma excelente ferramenta a ser } \\
\text { utilizada pelos cirurgiões-dentistas e pode } \\
\text { contribuir consideravelmente para o sucesso } \\
\text { profissional. Por outro lado, a falta de } \\
\text { conhecimento do tema em questão pode ocasionar } \\
\text { em resultados indesejáveis. }\end{array}$ \\
\hline $\begin{array}{l}\text { Simplício, } \\
2020\end{array}$ & $\begin{array}{l}\text { Dental press } \\
\text { journal of } \\
\text { orthodontics, }\end{array}$ & 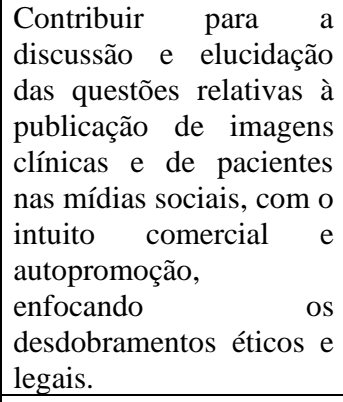 & $\begin{array}{l}\text { Revisão de } \\
\text { Literatura } \\
\text { Integrativa }\end{array}$ & $\begin{array}{l}\text { As mídias/redes sociais são valiosas ferramentas } \\
\text { de divulgação e troca de informação, por sua } \\
\text { agilidade, caráter democrático e baixo custo. } \\
\text { Contudo, sua utilização de forma abusiva e } \\
\text { enganosa, com infrações éticas e legais, associada à } \\
\text { dificuldade de fiscalização, pode trazer sérios } \\
\text { prejuízos, resultando em jurisprudências } \\
\text { desfavoráveis. }\end{array}$ \\
\hline Farsi, 2021 & $\begin{array}{c}\text { J Med Internet } \\
\text { Res }\end{array}$ & $\begin{array}{l}\text { Lançar luz sobre o uso da } \\
\text { mídia social em todo o } \\
\text { mundo e discutir como } \\
\text { ele tem sido usado como } \\
\text { uma ferramenta essencial } \\
\text { no setor de saúde na } \\
\text { perspectiva dos } \\
\text { profissionais de saúde }\end{array}$ & $\begin{array}{l}\text { Revisão de } \\
\text { literatura } \\
\text { integrativa }\end{array}$ & $\begin{array}{l}\text { As mídias sociais podem ser usadas não apenas } \\
\text { para melhorar o atendimento direto ao paciente, } \\
\text { mas também para aumentar o conhecimento do } \\
\text { público, facilitar a pesquisa, conectar profissionais, } \\
\text { melhorar a educação médica e combater crises de } \\
\text { saúde pública. No entanto, é fundamental encontrar } \\
\text { o equilíbrio certo entre os cuidados de saúde } \\
\text { digitais e tradicionais. Como a mídia social é um } \\
\text { fenômeno relativamente recente, mais pesquisas } \\
\text { são necessárias para determinar sua eficácia em } \\
\text { longo prazo e identificar as melhores estratégias } \\
\text { para maximizar suas vantagens e limitar os riscos. }\end{array}$ \\
\hline
\end{tabular}

Fonte: Autores.

Percebe-se pelos dados expostos no Quadro 1, que a literatura é convergente no que se diz respeito ao uso do marketing digital, especialmente quando este é pautado pela ética odontológica. 


\section{Discussão}

Após análise dos 5 estudos primários observou-se que à medida que o mundo continua seu avanço tecnológico, a mídia social acompanha, se tornando uma parte crucial na vida de bilhões de pessoas diariamente, afetando todos os setores imagináveis e isso não seria diferente com a indústria de saúde. O uso das ferramentas digitais na implementação do marketing odontológico tem evoluído de forma crescente, pois além de facilitar a interação profissional-paciente, é um método simples e eficaz para interação social, captação e fidelização de clientes (Lira et al, 2018).

Nos últimos anos, as redes sociais têm recebido grande atenção na área da saúde por profissionais e pesquisadores da área e quanto mais digitalmente orientado o mundo se torna, mais a mídia social é vista como um canal importante para promoção de saúde, emprego, recrutamento de novos pacientes, construção de uma marca forte e um marketing certeiro. (Farsi, 2021). O Brasil é líder em tempo de acesso às mídias sociais na América Latina. A audiência multiplataforma das redes sociais no Brasil é maior que a soma das da Argentina, México, Colômbia e Chile. Visto isso, a eficiência das mídias para interações com pacientes e colegas, bem como recursos e ferramentas de marketing para disseminar e acessar o conhecimento científico, é indiscutível (Simplício, 2020).

Por meio da mídia social, os cirurgiões-dentistas podem divulgar suas trajetórias, processo criativo, técnicas de tratamento, além de disseminar assuntos que eduquem a população em geral. O marketing de mídia deve ser um processo dinâmico, de contínua construção e criação de mercado de alta qualidade que se apoie no respeito geral e na ética interprofissional e dentista-paciente (Magalhães et al, 2018).

Os aspectos éticos são muito importantes quando se discute estratégias de marketing odontológico digital. Os temas mais discutidos em relação à aplicação ética da Internet e das tecnologias de comunicação na área da saúde são privacidade do paciente, confidencialidade e anonimato, segurança de dados, concorrência leal e propaganda real. No entanto, os princípios de ética e o código de conduta profissional de cada país devem primeiro ser respeitados (Meira et al, 2021).

Por isso, a ética, sendo um conjunto de valores e princípios que possibilitam a interação harmoniosa e respeitosa entre diferentes pessoas desconsiderando interesses individualistas e enganosos, deve ser aplicada em sua totalidade de intervenções de marketing para benefício do empreendimento odontológico e dos pacientes (Meira et al, 2021).

Guedes et al. (2021) afirmam também que o uso do marketing na prática odontológica é um fator determinante. O qual, o sucesso do cirurgião-dentista pode estar intimamente ligado, entretanto o profissional dever utilizar de forma correta, sempre seguindo o que é considerado no Código de Ética Odontológico para que não torne prejudicial para nenhuma das partes.

Em janeiro de 2019, o Conselho Federal de Odontologia apresentou a Resolução 196/2019, a qual alterou o Capítulo XVI do Código de Ética do Odontólogo. Ela permite a divulgação de autorretratos e imagens de "antes e depois" dos tratamentos odontológicos, com a autorização prévia do paciente ou do seu representante legal. Essa nova alteração, auxilia o cirurgião dentista no uso do marketing não infringindo o código ética. Sendo assim, vários usos possíveis para as redes e redes sociais online não infringem a lei nem desrespeitam os princípios éticos e considerando, portanto, a flexibilidade, amplo alcance, facilidade de uso e baixo custo, quando comparados aos meios tradicionais, desistir de usá-los ou se opor ao seu uso seria imprudente (Meira et al, 2021).

\section{Conclusão}

Desse modo, conclui-se que o marketing digital contribui para o sucesso do cirurgião dentista no seu cotidiano, podendo contribuir consideravelmente para o sucesso profissional dele, pois é uma maneira de disseminar conhecimentos, serviços e facilitar a comunicação e fidelização entre cliente-profissional. O marketing digital deve ser utilizado sempre da 
forma correta, seguindo o Código de Ética Odontológico, sendo de grande importância o conhecimento do último pelo cirurgião dentista. Através da realização dessa pesquisa, observou-se de forma clara que o assunto necessita de maior abrangência e visibilidade por meio das instituições de ensino, sendo ideal a abordagem do mesmo durante o período de graduação, para que dessa forma, a formação de profissionais engajados e conscientes das estratégias éticas sobre marketing digital seja efetiva, aumentando assim o número de sucesso profissional dos novos cirurgiões dentistas e, consequentemente, causando baixa nas fraudes e intercorrências éticas pertinentes ao assunto. Mais estudos são necessários para que o impacto do marketing digital pautado na ética profissional para os consultórios odontológicos seja avaliado, especialmente após os sombrios tempos da pandemia da COVID-19.

\section{Referências}

Aboalshamat, K., Alkiyadi, S., Alsaleh, S., et al. (2019). Attitudes toward social media among practicing dentists and dental students in clinical years in Saudi Arabia. The Open Journal Dentistry, 13, 143-149. 10.2174/1874210601913010143.

Ayres Pinto, D. J., \& Moraes, I. (2020). Digital media as a tool of manipulation in democratic electoral processes: An analysis of the brexit case. Revista de Estudios Sociales, (74), 71-82.

Brasil. Ciência e Tecnologia. Pesquisa revela que mais de 100 milhões de brasileiros acessam a internet. Disponível em: http://www.brasil.gov.br/ciencia-etecnologia/2016/09/pesquisa-revela-que-mais-de-100-milhoes-de-brasileiros-acessam-a-internet.

Conselho Federal de Odontologia. Resolução No 196 de 29 de janeiro de 2019. Rio de Janeiro: CFO; 2019. http://www.cfo.org.br.

de Lira, A. D. L. S., \& Magalhães, B. M. (2018). Digital marketing in dentistry and ethical implications. Brazilian Dental Science, $21(2), 237-246$.

Favaretto, M., Shaw, D., De Clercq, E., Joda, T., \& Elger, B. S. Big Data and Digitalization in Dentistry: A Systematic Review of the Ethical Issues. Int J Environ Res Public Health. 2020;17(7):2495. 10.3390/ijerph17072495.

Farsi, D. Social Media and Health Care, Part I: Literature Review of Social Media Use by Health Care Providers. J Med Internet Res. 23(4):e23205. 10.2196/23205. PMID: 33664014; PMCID: PMC8056296.

Galvão, M. C. B., \& Ricarte, I. L. M. (2019). Revisão sistemática da literatura: conceituação, produção e publicação. Logeion: Filosofia da informação, 6(1), 57-73.

Garbin, C. A. S., Ortega, M. M., Garbin, A. J. I., \& Saliba, T. A. (2018). O uso das redes sociais na odontologia: uma análise dos aspectos éticos de páginas de clínicas odontológicas. Rev Bras Odontol LegRBOL.5(1), 22-29. https://doi.org/10.21117/rbol.v5i1.135

Guedes, E. D. P. R., Borges, A. G., Gomes, C., Miasato, J. M., \& Gonçalves, S. S. (2021). Avaliação do conhecimento de estudantes de odontologia sobre marketing digital. Revista Rede de Cuidados em Saúde, 15(1).

Gross, D., Gross, K., \& Wilhelmy, S. Digitalization in dentistry: ethical challenges and implications. Quintessence Int. 2019;50(10):830-838. 10.3290/j.qi.a43151.

Kenny, P., \& Johnson, I. G. (2016). Social media use, attitudes, behaviours and perceptions of online professionalism amongst dental students. British dental journal, 221(10), 651-655. https://doi.org/10.1038/sj.bdj.2016.864

Magalhães, L. V, Frecalde T. S., Coltri, M. V, Barbosa, H. F, Guimarães, M. A, \& Silval. R. H. A. (2018). Publicidade odontológica e médica: análise comparativa das normas profissionais. $R G O, 66(2), 166-171$.

Martins, Y. V. M., Dias, J. N., \& Lima, I. P. C. (2018). A evolução da prática odontológica brasileira: revisão da literatura. Rev. Nova esperança.16(3), 83-90.

Meira, T. M., Prestes, J., Gasparello, G. G., Antelo, O. M., Pithon, M. M., \& Tanaka, O. M. (2021). The effects of images posted to social media by orthodontists on public perception of professional credibility and willingness to become a client. Progress in Orthodontics, 22(1), 1-8.

Oliveira, W. H. T., Prado, M. M., \& Silva, R. F. (2014). Publicidade odontológica na internet: os sites de compra coletiva. Rev Bras Odontol Leg RBOL. 1(1), 78-91.

Pereira, C. A. Dentistry and the social media. (2017). RGO - Revista Gaúcha de Odontologia, 65(3), 229-236.: <https://doi.org/10.1590/1981863720170002000073487>. https://doi.org/10.1590/1981-863720170002000073487.

Rocha, G. C. D., \& Souza Filho, V. B. (2016). Da guerra às emoções: história da internet e o controverso surgimento do Facebook. Encontro Regional Norte de História da Mídia, (4).

Simplício, A. H. D. M. (2020). Social media and Dentistry: ethical and legal aspects. Dental press journal of orthodontics, $24,80-89$.

Zilber, S., Monken, S., \& Quevedo-Silva, F. (2019). Adoção de mídias sociais por pequenas e médias empresas de saúde. BBR. Brazilian Business Review, 16, 453-469. 